\title{
Comparative Evaluation of the Effect of Different Endodontic Irrigation Protocols on the Microhardness of Root Canal Dentin: An In Vitro Study
}

\author{
Kavneet Takhar ${ }^{1}$ Neetu Jindal ${ }^{1}$ Renu Agarwal ${ }^{1}$ Megha Rani ${ }^{1}$ Shallu Bansal ${ }^{2}$ \\ ${ }^{1}$ Department of Conservative Dentistry and Endodontics, Surendera \\ Dental College and Research Institute, Sri Ganganagar, Rajasthan, India \\ 2 Department of Oral and Maxillofacial Surgery, Swargiya Dadasaheb \\ Kalmegh Smruti Dental College and Hospital, Nagpur, Maharashtra, \\ Address for correspondence Kavneet Takhar, MDS, Department of \\ Conservative Dentistry and Endodontics, Surendera Dental College \\ and Research Institute, Sri Ganganagar 335001, Rajasthan, India \\ (e-mail: kavneet.thakar@gmail.com).
} India

Dent J Adv Stud 2021;9:128-132.

\begin{abstract}
Keywords

- CHX

- EDTA

- HEBP

- microhardness

- propolis

Aim and Objective The present in vitro study was done to comparatively evaluate the effect of different endodontic irrigation protocols on the microhardness at coronal, middle, and apical levels of root canal dentin.

Method The total sample size for the study was 100 . Each sample consisted of a longitudinally sectioned half of a root of a single-rooted tooth, which was then embedded in acrylic resin. The prepared samples were divided randomly into five groups of twenty samples each. Each group was treated with its respective irrigant to be tested. Group I was the control-the specimens were treated with distilled water. The specimens in group II were treated with sodium hypochlorite $(\mathrm{NaOCl})$, followed by ethylenediamine tetra-acetic acid (EDTA). Specimens in group III were treated with $\mathrm{NaOCl}$, followed by $\mathrm{CHX}$. Specimens in group IV were treated with $\mathrm{NaOCl}$, followed by hydroxyethylidene bisphosphonate (HEBP), and specimens in group $V$ were treated with $\mathrm{NaOCl}$, followed by propolis. Following this, all the samples were placed on the Vickers microhardness tester, and the results were tabulated and statistically analyzed to determine the irrigant solutions' effect on the microhardness of root dentin at coronal, middle, and apical third.

Results Intergroup comparison of various irrigants showed that 18\% HEBP showed the least reduction in the microhardness values of root canal dentin, and 17\% EDTA caused the maximum decrease in microhardness values with a significant difference. When the baseline values were compared with the values after the use of experimental solutions in all the groups, it was observed that the microhardness gradually decreased from coronal to apical third of root dentin.

Conclusion On the basis of the results obtained, it can be concluded that weak chelators such as $18 \%$ HEBP do not affect the microhardness considerably, and that they can be used in future in place of strong chelators, that is, EDTA, which make root dentin weak by more microhardness reduction.
\end{abstract}

published online October 18, 2021
DOI https://doi.org/ 10.1055/s-0041-1736262. ISSN 2321-1482.

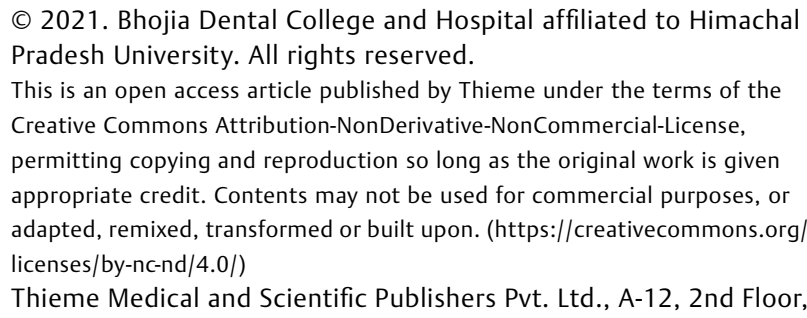

(c) 2021. Bhojia Dental College and Hospital affiliated to Himachal Pradesh University. All rights reserved.

This is an open access article published by Thieme under the terms of the Creative Commons Attribution-NonDerivative-NonCommercial-License, permitting copying and reproduction so long as the original work is given appropriate credit. Contents may not be used for commercial purposes, or adapted, remixed, transformed or built upon. (https://creativecommons.org/ licenses/by-nc-nd/4.0/)

Thieme Medical and Scientific Publishers Pvt. Ltd., A-12, 2nd Floor, Sector 2, Noida-201301 UP, India 


\section{Introduction}

Irrigation protocol of the root canal system facilitates in complete debridement, adequate lubrication, microbial destruction, and tissue dissolution, and helps in cleaning areas that are inaccessible during mechanical cleansing. Apart from beneficial effects, irrigants may exhibit detrimental effects on the dentin or root canal filling materials. Reports have indicated that the use of popular irrigants ethylenediamine tetra-acetic acid (EDTA) and sodium hypochlorite $(\mathrm{NaOCl})$ can demonstrate erosion of canal walls. ${ }^{1}$

The recently introduced irrigant hydroxyethylidene bisphosphonate (HEBP) also known as etidronate, shows lesser chelating ability, which causes minimal modifications in the flexural strength of mineralized dentin and hydroxyapatite/collagen ratio. An alternate irrigation protocol with HEBP has been suggested as a replacement to EDTA, because of its mild chelating ability. ${ }^{2}$ Propolis, another new naturally occurring irrigant, has also not been researched adequately for its effect on microhardness of root canal dentine.

The purpose of this study was to evaluate and compare the effect of four different irrigants on the microhardness of root dentine at coronal, middle, and apical third levels using a Vickers microhardness tester.

\section{Materials and Method}

\section{Sample Preparation}

Fifty recently naturally extracted human single rooted teeth were collected, and their decoronation was done at the cementoenamel junction with the help of a diamond disc at low speed. The root segments were taken as samples for study, which were longitudinally sectioned in buccolingual direction with the help of diamond disc at low speed (NSK) to obtain 100 sample size. Following this, root segments were embedded in autopolymerizing acrylic resin horizontally, leaving canals' dentin surface exposed. Then, surface of the mounted root samples were refined and smoothed to remove any surface scratches with the help of ascending grades of carbide abrasive papers $500,800,1000$, and 1200 grit under distilled water and further polished with alumina suspension.

Microhardness values were measured for each sample at coronal, middle, and apical third using Vickers microhardness tester (type: 7005) at magnification of $\times 100$ under $200 \mathrm{gm}$ load for 30 seconds to get the baseline values (-Fig. 1).

\section{Samples Treatment}

The samples were categorized into five groups $(n=20)$. All groups were treated by immersion method with their respective irrigants, that is, 17\% EDTA (Neelkanth Health Care, India), 2\% chlorhexidine (CHX) (Neelkanth Health Care, India), 18\% HEBP (Micro Hydrochem Pvt Ltd., Mumbai, India), 4\% Propolis (Forever Bee Propolis, Aloe Vera of America Inc.), 3\% $\mathrm{NaOCl}$ (Organo Biotech Laboratories Pvt. Ltd, India), and one group (control group) with saline (Denis Chem Laboratory, India), as per the following protocol:
- Group I: (control group) Samples treated with saline for 5 minutes. This group served as control group.

- Group II: Samples treated with 3\% $\mathrm{NaOCl}$ for 5 minutes, followed by 17\% EDTA for 5 minutes.

- Group III: Samples treated with 3\% $\mathrm{NaOCl}$ for 5 minutes, followed by $2 \%$ CHX for 5 minutes.

- Group IV: Samples treated with $5 \% \mathrm{NaOCl}$ for 5 minutes, followed by $18 \%$ HEBP for 5 minutes.

- Group V: Samples treated with $5 \% \mathrm{NaOCl}$ up to 5 minutes, followed by $4 \%$ Propolis for 5 minutes.

After the samples were treated with irrigants, postapplication value of Vickers microhardness (Vickers hardness number [VHN]) was measured at the surface of the dentin of each sample using the same parameters as done for the baseline values (-Fig. 2). Statistical analysis was done, and the readings obtained were subjected to one-way analysis of variance (ANOVA) and Tukey's test.

\section{Results}

Intergroup comparison of various irrigants showed that $18 \%$ HEBP showed the least reduction in the microhardness values of root canal dentin, and 17\% EDTA caused the maximum decrease in microhardness values with a significant difference $(\boldsymbol{p}<0.00)$ ( - Table 1). When the baseline values were compared with the values after the use of experimental solutions in all the groups, it was observed that the microhardness gradually decreased from coronal to apical third of root dentin (-Table 2 ).

\section{Discussion}

Irrigation in endodontic treatment has always played a pivotal role The flushing mechanism of various irrigants used during and after instrumentation facilitates removal of tissue remnants, microorganisms, and dentinal chips from the root canal. Few irrigating solutions like EDTA dissolve either inorganic or organic tissue of the root canal by their chelation action, which drastically reduces the microhardness of dentin, thus weakening the dentin morphology. ${ }^{3}$ The present study evaluated the effects of different endodontic irrigants on the microhardness of root canal dentin. The various irrigants used were $2 \% \mathrm{CHX}$, a traditional chelator, that is, $17 \%$ EDTA, a newer chelating agent, that is, $18 \%$ HEBP, a modern herbal irrigant, that is, $4 \%$ Propolis in combination with $\mathrm{NaOCl}$, as it is recommended for an effective irrigation regimen.

Zaccaro et al determined the efficacy of 17\% EDTA, $3 \% \mathrm{H}_{2} \mathrm{O}_{2}$, and $10 \%$ citric acid under scanning electron microscopy (SEM). The mean number of visible open dentinal tubules and the largest number of visible tubules were present in the coronal third, followed by the middle third, while in the apical third, the least number of open dentinal tubules were found, thereby resulting in deeper permeability of the solution for removal of the smear layer from dentinal tubules in coronal third and lesser permeability in apical third. ${ }^{4}$ 
Table 1 Mean and SD values of microhardness (VHN) of different groups after irrigating it with various irrigation solutions at coronal, middle, and apical level

\begin{tabular}{|l|l|l|l|l|}
\hline \multicolumn{2}{|l|}{ Groups } & Coronal & Middle & Apical \\
\hline \multirow{2}{*}{ Saline (control) (group I) } & Mean & 0.120 & 0.130 & 0.105 \\
\cline { 2 - 5 } & SD & 0.1508 & 0.1895 & 0.3069 \\
\hline \multirow{2}{*}{ EDTA (group II) } & Mean & 10.430 & 0.710 & 0.115 \\
\cline { 2 - 5 } & SD & 0.9948 & 0.4051 & 0.3066 \\
\hline \multirow{2}{*}{ CHX (group III) } & Mean & 7.120 & 0.260 & 0.105 \\
\cline { 2 - 5 } & SD & 0.5644 & 0.3050 & 0.4032 \\
\hline \multirow{2}{*}{ HEBP (group IV) } & Mean & 1.095 & 0.480 & 0.155 \\
\cline { 2 - 5 } & SD & 0.5753 & 0.4697 & 0.3927 \\
\hline Propolis (group V) & Mean & 2.320 & 0.455 & 0.250 \\
\cline { 2 - 5 } & SD & 0.6288 & 0.4430 & 0.6947 \\
\hline
\end{tabular}

Abbreviations: CHX, chlorhexidine; EDTA, ethylenediamine tetra-acetic acid; HEBP, hydroxyethylidene bisphosphonate; SD, standard deviation; VHN, Vickers hardness number.

Table 2 Intergroup comparison of microhardness (VHN) of dentin at coronal, middle, and apical third of teeth after using different irrigation protocols using analysis of variance (ANOVA)

\begin{tabular}{|c|c|c|c|c|c|c|}
\hline & & Sum of squares & df & Mean square & $F$ & $p$ \\
\hline \multirow[t]{3}{*}{ Coronal } & Between Groups & 1543.194 & 4 & 385.798 & 937.662 & $0.000^{\mathrm{a}}$ \\
\hline & Within groups & 39.088 & 95 & 0.411 & & \\
\hline & Total & 1582.281 & 99 & & & \\
\hline \multirow[t]{3}{*}{ Middle } & Between Groups & 3.956 & 4 & 0.989 & 6.964 & $0.000^{\mathrm{a}}$ \\
\hline & Within groups & 13.490 & 95 & 0.142 & & \\
\hline & Total & 17.445 & 99 & & & \\
\hline \multirow[t]{3}{*}{ Apical } & Between Groups & 0.304 & 4 & 0.076 & 0.385 & 0.819 \\
\hline & Within groups & 18.764 & 95 & 0.198 & & \\
\hline & Total & 19.068 & 99 & & & \\
\hline
\end{tabular}

Abbreviation: VHN, Vickers hardness number.

${ }^{\mathrm{a}}$ The mean difference is significant at the 0.05 level.

$17 \%$ EDTA, in the present study, caused reduction of dentin microhardness in coronal and middle third, and was comparatively not effective in dentin microhardness reduction from apical third, as shown by Zaccaro et al. The use of $17 \%$ EDTA, which caused disintegration of peritubular and intertubular dentin, resulted in substantial enlargement of the diameter of tubules, as there is greater tubular density near the pulp, and the area occupied by open tubules also increased to a high number. EDTA also removed inorganic content from interfibrillar portion of intertubular dentin, thus causing reduction in inorganic content of dentin and reduction in microhardness

After EDTA, 2\% CHX showed more impact on microhardness $7.12( \pm 0.56 \mathrm{VHN})$ coronally than in middle third 0.26 $( \pm 0.30 \mathrm{VHN})$ and apical third $0.10( \pm 0.40 \mathrm{VHN})$ of root canal. Its ability to penetrate the whole length of dentinal tubules and to dissolve tissues was lower as compared with other experimental groups.
2\% CHX irrigant has good antibacterial activity, substantivity, tissue-dissolving capability, and the capacity to inhibit the adherence of certain bacteria to dentine. ${ }^{5}$

Oliveira et al in their study assessed the impact of $2 \% \mathrm{CHX}$ and $1 \% \mathrm{NaOCl}$ on the root canal dentins' microhardness and inferred that $\mathrm{CHX}$ and $\mathrm{NaOCl}$ solutions effectively caused a microhardness reduction of root canal dentin from the pulpdentin interface. ${ }^{6}$

The result of the present study also showed that $3 \%$ $\mathrm{NaOCl}+2 \% \mathrm{CHX}$ displayed varied results in microhardness reduction only in coronal third. The reason for such an observation could be that number of mean visible open dentinal tubules and largest number of visible tubules were more in the cervical third, as compared with the middle and apical thirds, resulting in deeper penetration of the solution in dentinal tubules. The passive removal of smear layer after irrigating it with $2 \% \mathrm{CHX}$ and $3 \% \mathrm{NaOCl}$ from the coronal structure can be attributed to its open dentinal 


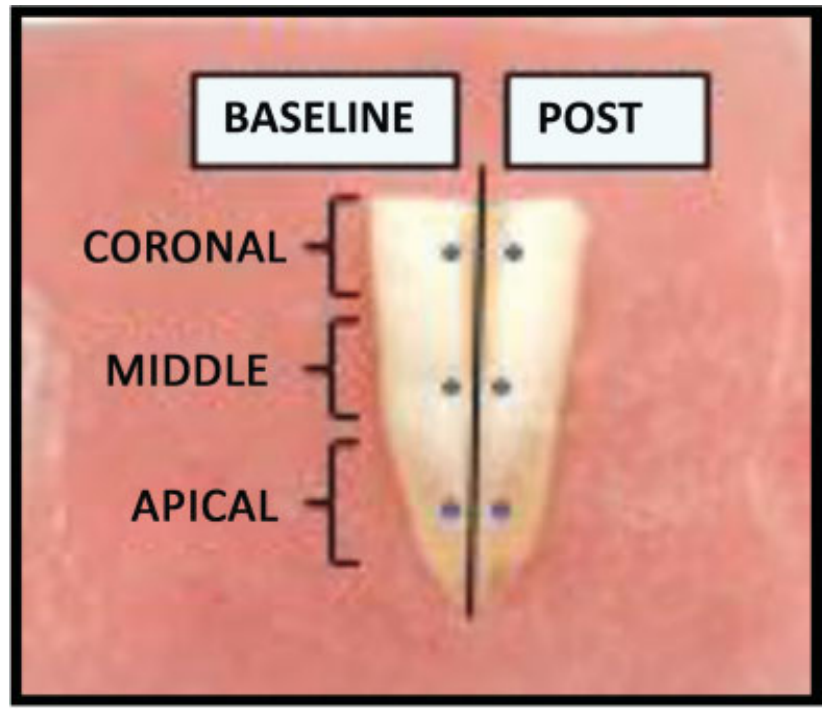

Fig. 1 Marking of reference point for baseline and post values on a longitudinally sectioned tooth.

tubules, leading to greater amount of microhardness reduction in coronal third.

However, contrary to our study, another study by Weshah et al, who investigated the effect of only $2 \% \mathrm{CHX}$ on the root dentins' microhardness at different level, showed no statistically significant reduction $(p>0.05)$ difference in the decrease of dentin hardness before or after application of $2 \%$ CHX in any root section. ${ }^{7}$

$18 \%$ HEBP, also known as etidronic acid, is a substance that is a selective chelator because of its minimal ramifications on the dentin structure and can even be used in combination with $\mathrm{NaOCl}$ without interfering with its antimicrobial property. ${ }^{1}$ Hulsmann et al stated that HEBP shows lesser chelating ability, which causes minimum alterations in the flexural strength and apatite/collagen ratio of mineralized dentin. ${ }^{2}$

Cobankara et al evaluated the chelation effect of 17\% EDTA, $18 \%$ HEBP, citric acid, and peracetic acid. He found out that $18 \%$ HEBP had the least effect on the microhardness than all other irrigants, as the mineral content of root canal dentin had shown less erosion and so it has a potential to be used as an alternative to EDTA. ${ }^{8}$

Dinesh et al compared the effect on microhardness of root canal dentin of 17\% EDTA, MTAD, and 18\% HEBP irrigating solutions. Even this study group treated with HEBP showed the highest reduction in microhardness reduction at coronal and middle level and least in apical third. This could be due to the greater intertubular dentin area availability at coronal followed by middle level and the least at apical third. He concluded that, when a mild chelating protocol using HEBP is subjected on the root dentin, there is greater chelation action in coronal and middle level, while the lower efficacy of HEBP at apical level can be attributed to sclerosed dentin. ${ }^{9}$

The result of present study are similar to the results of the above-mentioned studies wherein coronal third of the canal showed greater reduction in microhardness of root dentin with a significant difference $(p<0.00)$. Such a difference could be due to the increased depth of demineralization of

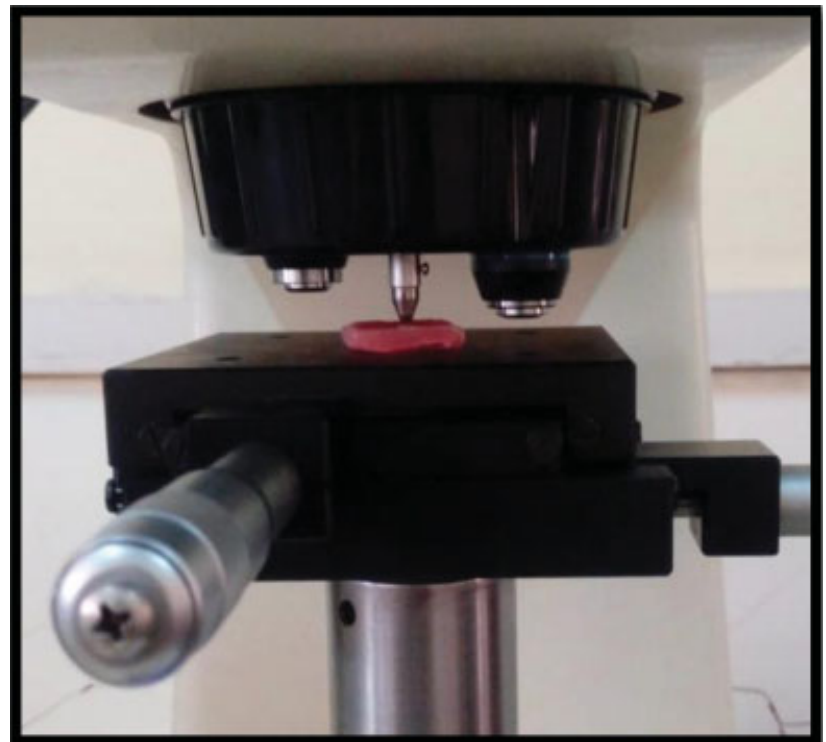

Fig. 2 Sample placed under the indenter.

EDTA in relation to HEBP, thus causing less erosion and alteration of dentin structure.

In this study, 4\% Propolis also led to the decrease in microhardness values at all the levels but was found to effect microhardness greatest in apical third $0.25( \pm 0.69 \mathrm{VHN})$.

Propolis chemical composition has proved that its highly variable and depends on the collection site's flora. The various groups present in propolis are aliphatic acids, aromatic acids, aromatic acid esters, flavonoids, and some triterpenoids. The presence of flavonoids and esters of phenolic acids might be the causative factor in the significant reduction of microhardness. ${ }^{10}$

Bhagwat et al comparatively evaluated 17\% EDTA, 2\% CHX, $18 \%$ HEBP, and $4 \%$ Propolis effects on the microhardness of root dentin. Even in this study, it was found that use of $4 \%$ Propolis caused a greater reduction in the root canal dentin microhardness but comparatively less in 17\% EDTA and $2 \%$ CHX. They concluded that if antimicrobial activity and effect on the root dentin microhardness were the only requirements of an endodontic irrigant, the results of this study would suggest that Propolis is equal to $\mathrm{NaOCl}^{1}$

Elgendy evaluated the comparative action on microhardness of $0.2 \%$ chitosan, $4 \%$ Propolis, $5 \% \mathrm{NaOCl}$, and $17 \%$ EDTA on human root dentin. He found out that significant decrease in the microhardness of root dentin was seen with $4 \%$ Propolis. He stated that it may be due to the phenolics group present in them, which has the ability of chelating metals. These weak acids could be adsorbed on hydroxyapatite molecules, and after adsorption, the reaction mechanism involves equilibrium reactions with the hydroxyapatite mineral content. ${ }^{11}$

The present study results are in accordance with the above study, where Propolis showed significant reduction in microhardness of dentin. 4\% Propolis showed promise as irrigants with lesser ramifications on the dentine mineral content. Further investigation into the depth of demineralization is imperative to provide greater and predictable data on the clinical performance of Propolis. 
Therefore, after analyzing the result of the study, it can be inferred that while considering root canal being different morphologically and subjected to the same irrigation regimen, it showed that the root thirds were affected differently, as microhardness of dentin decreased from coronal to apical third. 18\% HEBP was introduced as an endodontic irrigant, due to its outstanding conservative abilities, as it does not affect the microhardness of root dentin considerably, and it can be used in future in place of strong chelators, that is, EDTA, which makes root dentin weak by more microhardness reduction of root canal dentin.

\section{Conclusion}

Hence, on the basis of the results obtained, it can be concluded that weak chelators such as 18\% HEBP do not affect the microhardness considerably, and that they can be used in future in place of strong chelators, that is, EDTA, which make root dentin weak by more microhardness reduction.

\section{Conflict of Interest}

None declared.

\section{References}

1 Bhagwat SA, Lopez TA, Mandke LP. Comparison of the effect of ethylenediamine tetra-acetic acid, chlorhexidine, etidronic acid and propolis as an irrigant on the microhardness of root dentin: An in vitro study. J Dent Res 2016;3(01):23-30

2 Hülsmann M, Heckendorff M, Lennon A. Chelating agents in root canal treatment: mode of action and indications for their use. Int Endod J 2003;36(12):810-830

3 Paqué $\mathrm{F}$, Ganahl D, Peters OA. Effects of root canal preparation on apical geometry assessed by micro-computed tomography. J Endod 2009;35(07):1056-1059

4 Zehnder M, Schmidlin P, Sener B, Waltimo T. Chelation in root canal therapy reconsidered. J Endod 2005;31(11):817-820

5 Siqueira JF Jr, Paiva SS, Rôças IN. Reduction in the cultivable bacterial populations in infected root canals by a chlorhexidinebased antimicrobial protocol. J Endod 2007;33(05):541-547

6 Oliveira LD, Carvalho CA, Nunes W, Valera MC, Camargo CH, Jorge AO. Effects of chlorhexidine and sodium hypochlorite on the microhardness of root canal dentin. Oral Surg Oral Med Oral Pathol Oral Radiol Endod 2007;104(04):e125-e128

7 Al Weshah MM. The in vitro effect of $2 \%$ chlorhexidine on dentine hardness. PODJ 2011;31(01):173-177

8 Cobankara FK, Erdogan H, Hamurcu M. Effects of chelating agents on the mineral content of root canal dentin. Oral Surg Oral Med Oral Pathol Oral Radiol Endod 2011;112(06):e149-e154

9 Dineshkumar MK, Vinothkumar TS, Arathi G, Shanthisree P, Kandaswamy D. Effect of ethylene diamine tetra-acetic acid, MTAD, and HEBP as a final rinse on the microhardness of root dentin. J Conserv Dent 2012;15(02):170-173

10 Zare Jahromi M, Toubayani H, Rezaei M. Propolis: a new alternative for root canal disinfection. Iran Endod J 2012;7(03):127-133

11 Elgendy AA. Evaluation of comparative action of $0.2 \%$ chitosan, $4 \%$ propolis, $2.6 \% \mathrm{NaOCl}$ and $17 \%$ EDTA on human root dentin microhardness. Egypt Dent J 2017;63:1069-1075 